\title{
Modeling and Controlling Friendliness for An Interactive Museum Robot
}

\author{
Chien-Ming Huang, Takamasa Iio, Satoru Satake, Takayuki Kanda \\ Intelligent Robotics and Communication Laboratory, ATR \\ Email: \{cmhuang, iio, satoru, kanda\}@atr.jp
}

\begin{abstract}
Advances in robotic technologies have enabled interactive robots to utilize humanlike social behaviors to interact with people in public places such as museums. While these behaviors have shown promise in engaging people, they have been designed and applied to users uniformly. Humans, however, behave differently according to their relationships with others. Behavioral changes, from neutral to friendly, contribute to the development of interpersonal relationships. Friendliness, in particular, plays an important role in the early development of a relationship. In this work, we explore how an interactive robot might nonverbally express a variety of friendly behaviors in a museum scenario. Four behavioral variables-response time, approach speed, individual distance, and attentiveness - contributing to perceived friendliness were modeled and implemented for the interactive museum robot. The results of our study showed that people perceived the differences in the designed robot behaviors and related those differences to the friendliness of the robot to varying degrees. This work serves as a building block toward the development of human-robot relationships and has implications on designing friendly behaviors for interactive robots.
\end{abstract}

\section{INTRODUCTION}

[ With the advances in robotic technologies, robots have been deployed to interact with people in public places such as museums [9, 30, 32, 35]. To engage and build rapport with people, interactive museum robots have been designed to use verbal speech and nonverbal behaviors during exhibition guiding. Friendliness is particularly important to the formation of positive impressions and the key ingredient in the early development of rapport and relationship [40]. Prior work has shown that friendly behaviors, manifested in sounds and facial expressions, are important for an engaging museum robot [32].

While using engaging and friendly behaviors might have shown promise in improving people's visit experiences in the past, they were designed and applied uniformly to all visitors. However, human guides ordinarily change their behaviors gradually to match and form relationships with visitors. For example, in designing our study, we observed that a human museum guide gradually showed friendlier behaviors, such as verbally referring to previous interaction experiences, toward people who repeatedly visited the museum and were considered important visitors. Therefore, interactive museum robots should employ friendly behaviors accordingly to regulate and foster the development of relationships with users. For instance, a museum robot may exhibit friendlier behaviors towards the frequent, important visitors to build rapport and

The first author is now affiliated with the University of Wisconsin-Madison (cmhuang@cs.wisc.edu).

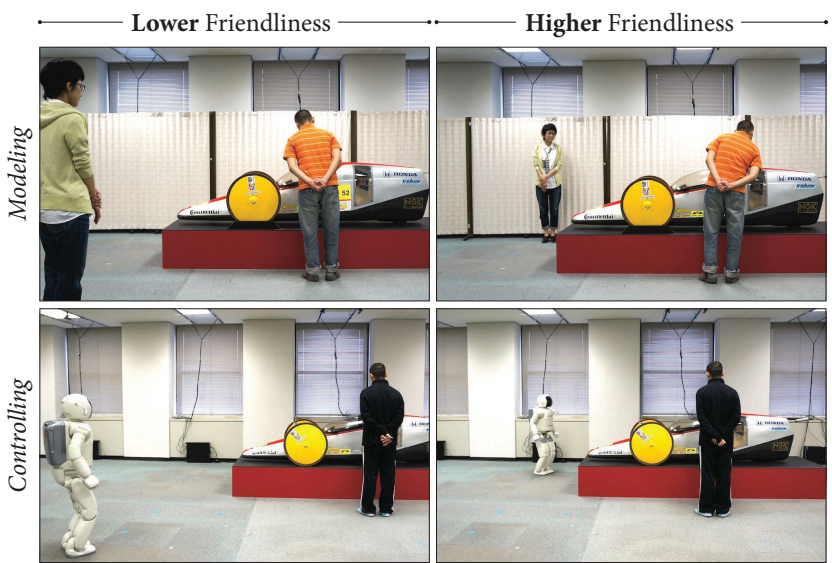

Fig. 1. We modeled how people might exhibit behavior differently to show various levels of friendliness (Top) and evaluated the model of friendliness in a human-robot interaction study (Bottom).

enhance their visit experiences. However, if the robot acts overly friendly towards a first time visitor, the visitor might feel intimidated and socially awkward.

In this paper, we aim to investigate how interactive robots might exhibit behaviors differently and leverage the differences in behavior to convey varying levels of friendliness that contribute to the formation of rapport and relationships [40]. We contextualized the investigation in a museum scenario in which an autonomous humanoid robot, acting as a tour guide, interacted with participants. Our investigation involved understanding how people might behave differently toward others according to their relationships (Top of Figure 11, building a behavioral model of varying friendliness for the humanoid robot, and evaluating the model in a human-robot interaction study to assess whether or not participants could perceive the differences in the robot's behaviors and how they related the differences to their perceptions of the robot (Bottom of Figure (1). Findings of our investigation provide insights into designing friendly robot behaviors to engage people and support the development of human-robot relationships.

\section{BACKGROUND}

\section{A. Human Behavior and Interpersonal Relationship}

Interpersonal relationships govern how humans use behavior during interaction. Humans do not use behavior uniformly toward other people whom they have different relationships with. For example, people exhibited different spatial behaviors, such as seating preferences, toward those whose status was 
higher, equal, or lower than themselves [25, 37] and maintained different interpersonal distances while interacting with those whom they had different friendships with [17, 19, 43].

Moreover, gradual changes in behavior are closely related to the course of relationship development. The development of interpersonal relationships has been conceptualized as a layered construct and described as a process of social penetration [2]. It involves (1) exchanges of interpersonal verbal and nonverbal behaviors and (2) subjective assessments associated with the behaviors to evaluate a person and the interaction with that person. As the relationship grows, the process of social penetration moves from superficial, outer layers to intimate, inner layers, resulting in changes in uses of behaviors, such as becoming more behaviorally open and accessible by maintaining greater eye contact.

Through the process of forming relationships, a feeling of rapport emerges between individuals. In particular, friendliness is key to the formation of positive impressions and the early development of rapport and relationship [40]. For instance, in service encounters, service providers utilize nonverbal behavior to show friendliness and build rapport with their customers, leading to improved perceptions of service quality [38]. While friendly behavior plays a key role in building rapport and developing a relationship, it is important to employ appropriately friendly behaviors. Indeed, the ability to display friendly behaviors that match the corresponding interpersonal relationship has developmental significance. Indiscriminate friendliness describes the lack of this ability to apply friendly behaviors in an appropriate way during interaction [41]. For example, young children with indiscriminate friendliness display overly friendly behavior when interacting with strangers.

Friendliness is communicated through multiple behavioral channels. Individuals draw on a variety of immediacy behaviors - aiming to reduce psychological distances with othersto build rapport and increase levels of friendliness [27]. Immediacy behaviors, such as standing closer, making more eye contact, and orienting more directly, present more opportunities for immediate interaction to gain affiliative relationships and desirable outcomes. For example, people use a shorter distance to earn approval [31], gain compliance [16, 33], and increase perceived persuasiveness [1].

Proxemic behaviors, concerning individuals' use of space, are one of the most effective nonverbal behaviors to signal interpersonal relationships. Hall placed individuals' use of distance in interaction into four layers-intimate, personal, social, and public distance [17]. This layer structure is analogous in structure to the process of social penetration. The use of distance is regulated by people's attitudes and relationships with others [18] and is used as a measure of friendship [28].

To build affiliative relationships and have affective interactions with their users in public places such as museums, robots need to communicate friendliness effectively. Nonverbal behaviors are particularly effective for affective communication [5] and a key constituent of the feeling of rapport in individuals [40]. In this work, we aim to explore the design space of how robots might nonverbally communicate friendliness to varying degrees, serving as a building block for the development of human-robot relationships.

\section{B. Robot Behavior and Human-Robot Relationship}

To enable effective and natural interactions between humans and robots, robotics researchers have drawn on models of human interaction and literature in human communication to equip interactive robots with a repertoire of social behaviors including gaze, gestures, proxemic behaviors, and more [21, 22, 23, 29, 39]. A robot's behavior affects how people would interact with and perceive it. For instance, a robot's gaze cues were shown to regulate how people would distance themselves from the robot [29]. While appropriately designed social behaviors have been shown to be effective in eliciting desirable outcomes, such as preferred perceptions of the robot, it is unclear whether or not applying these behaviors uniformly to people meant to interact with the robot repeatedly in a long period of time would yield similar outcomes.

As in human interaction where people change their preferences and acceptance of others' behavior according to their relationships [2, 3], people also show similar changes in interacting with robots. It has been shown that people who self-reported previous experience with robots accepted a robot's position at a shorter individual distance [39]. Moreover, research further demonstrated that participants' preferences for a robot's approach distance changed over a five-week investigation [24]. As the investigation progressed, participants accepted a shorter interaction distance with the robot, especially when the robot's appearance was humanlike. These changes may be due to the participants developing a relationship with the robot, suggesting that people preferred when the robot's behavior matched their perceived relationship as time went on.

To facilitate the development of relationships with their users, robots should apply behaviors adaptively in the course of a relationship as a way of building rapport and communicating affective states such as friendliness. Similar to the social penetration in interpersonal relationships, the relationships between humans and robots are formed as behaviors change from detached, neutral to close, friendly.

\section{MODELING FRIENDLINESS FOR A RoBOT}

This section presents the process of building a behavioral model of varying friendliness. This process involves (1) collecting data on manipulated behaviors in inducing varying perceived friendliness, (2) identifying key behavioral variables that contribute to the induced perceptions, and (3) extracting parameters for those behavioral variables from the data.

\section{A. Data Collection}

We designed a museum scenario in which participants acted as an exhibition guide and a male confederate acted as a visitor (Figure 2). We manipulated the participants' behavior towards the confederate by asking them to behave as if they were guiding a visitor of three different status as of low, medium, or high importance (cf. [25, 37]). A similar approach to manipulating participants' behavior by manipulating their attitudes toward 


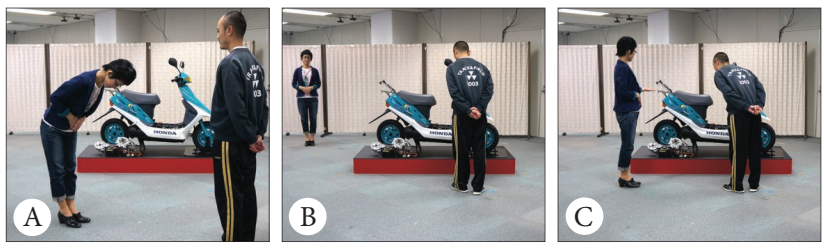

Fig. 2. Data collection study: Participants acted as a museum guide while a confederate acted as a visitor. Participants could approach the confederate to greet him (A), waited while the confederate attended to the exhibit (B), and could approach again to present the exhibit (C).

the other was used previously to study interpersonal distance [2, 26, 31]. In addition, we manipulated the confederate's gaze behavior. In one case (gaze cuing), the confederate gazed at the participant while attending to the exhibit. In the other case (without gaze cuing), the confederate attended to the exhibit without making eye contact with the participant. This gaze manipulation represented two typical gaze behaviors in an exhibition setting. Gaze cues have been shown to affect human spatial behavior [4, 29], a major part of interaction in a museum setting.

To ensure that the manipulation of the participants' attitude toward the confederate successfully elicited different behaviors and to understand how these behaviors were related to perceived friendliness of the participants, three raters, two native Japanese speakers and one native English speaker blind to the manipulation, rated the friendliness of the participants using a seven-point scale. Inter-rater reliability analysis using interclass correlations (ICC) [36] revealed high correlations between the three raters $(\operatorname{ICC}(3,3)=.927)$. We further used a two-way fixed-effects analysis of variance (ANOVA), using the manipulated attitude and the confederate's gaze cues as fixed factors, to analyze the perceived friendliness of the participants. The manipulation of the participants' attitude had a significant effect on the perceived friendliness, $F(2,78)=$ 198.07, $p<.001, \eta_{p}^{2}=0.492$. A post-hoc Tukey's HSD test further showed that the raters rated participants who guided the confederate of high status to behave in a significantly more friendly manner than those guiding the confederate of medium status, and participants guiding the confederate of medium status were perceived to behave in a significantly more friendly manner than those guiding the confederate of low status. These results suggested that participants used behaviors of different levels of perceived friendliness in interacting with the confederate they thought having different relationships with.

The participants were instructed to stand next to the exhibit while waiting for the visitor to arrive. Once the visitor entered the exhibition room, the participants could approach the visitor and greet him (Figure 2.A). After greeting him, participants moved to a waiting position while the visitor went to see the exhibit (Figure 2 B). Then, participants could approach the visitor to present the exhibit (Figure 2 C). After presenting the exhibit, participants moved to a waiting position and waited for the visitor to leave the exhibition room. Note that whether or not to approach and greet the confederate and present the exhibit was the participant's choice. This scenario included two typical interaction events-greeting and presenting-in a

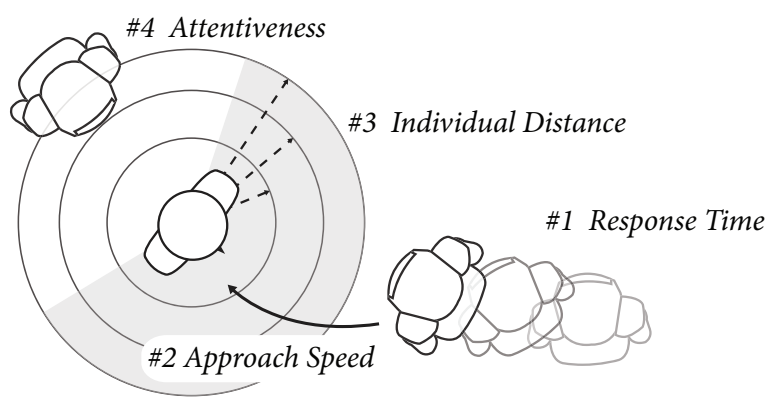

Fig. 3. The four behavioral variables-response time, approach speed, individual distance, and attentiveness - that encode perceived friendliness.

museum setting. The confederate was trained to behave the same regardless of the participants' behavior.

Fourteen participants (six females and eight males) ranging from 19 to 23 years in age $(M=20.90, S D=1.29)$ were recruited for this study. All participants were native Japanese speakers and were compensated 3000 Japanese yen for their participation. In addition to a video recording of the interaction, both the participants' and confederate's walking trajectories were recorded using a tracking system for behavioral analysis. Each participant enacted the role of exhibition guide for the six combinations, yielding 84 interaction episodes.

\section{B. Interaction Observation}

From the collected data, we observed that participants displayed behaviors differently when interacting with the confederate of different relationships, leading to varying levels of perceived friendliness. These observations were consistent with the literature in interpersonal relationships [2, 14]. In particular, we observed that the participants used different interpersonal distance and showed varying levels of readiness to interact when guiding the confederate of different relationships. It appeared that participants who stood closer to and readily displayed an intention to interact with the confederate were perceived to show greater friendliness. Another observation was that participants of different perceived friendliness chose different areas to wait after greeting and presenting.

\section{Behavioral Variables}

From the above observations, we identified four behavioral variables-response time, approach speed, interpersonal distance, and attentiveness- that differentiated perceived friendliness (Figure 3 ) and considered the four variables for both greeting and presenting behaviors in the following analyses.

Response time was defined as the time difference between the trigger action from the confederate and the response action from the participant. For the greeting behavior, it was defined as the difference between when the confederate entered the exhibition room and when a participant started to approach to greet him. For the presenting behavior, it was further defined by the manipulation of confederate's gaze behavior. In the case of no gaze cuing, the response time was measured by the difference between when the confederate stopped in front of the exhibit and when the participant started to approach him. In the case of gaze cuing, it was measured by the difference 


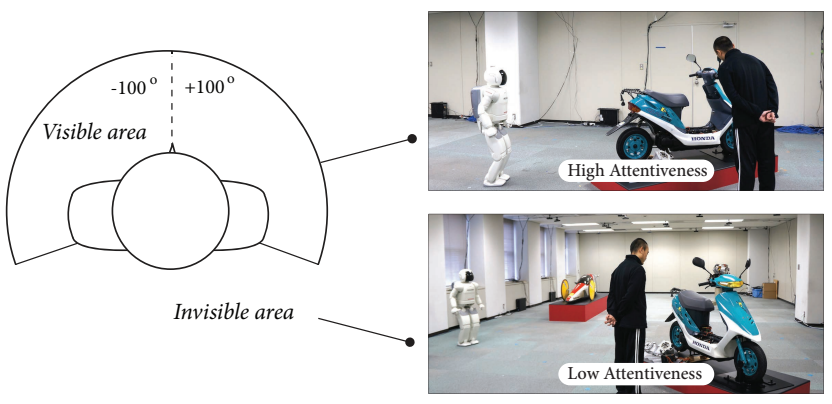

Fig. 4. Attentiveness is determined by the location at which the guide waits while the visitor is attending to an exhibit. Attentiveness is high when the guide waits inside the visitor's visible area.

between when the confederate gazed at the participant and when the participant started to approach. We considered this variable contributing to the perceived friendliness because responsiveness has been suggested to support the formation of rapport and the development of relationship [40].

Approach speed was calculated as the distance between the beginning and end position of approach, divided by the travel time of the movement. Individual distance was the distance between the participant and the confederate during the event of greeting and presenting. Attentiveness was determined by the location at which the participants chose to wait. We categorized waiting locations into two cases. One is within the visitor's visible area, representing high attentiveness, while the other one is outside of the visitor's visible area, representing low attentiveness (Figure 4).

These four behavioral variables, operating in the temporal (i.e., response time and approach speed) and spatial (i.e., individual distance and attentiveness) domains, contribute to the construct of openness and accessibility in communication [2]. In the following paragraphs, we verify whether or not participants' behaviors, manifested in the four variables, differed significantly when they were related to various friendliness. We used a two-way fixed-effects analysis of variance (ANOVA), using the manipulated attitude and the confederate's gaze behavior as fixed factors, to analyze response time, approach speed, and individual distance. A chi-square test of independence was used to analyze participants' attentiveness.

\section{Greeting Behavior}

Participants of low perceived friendliness did not approach the confederate to greet him. Thus, they were excluded from this analysis. For all the measures there was no main effect of the confederate's gaze behavior. Hereafter, we focus on the effect of the manipulated attitude on behaviors of varying perceived friendliness.

1) Response Time (s): Participants of high perceived friendliness responded significantly faster $(M=0.40, S D=0.42)$ than those of medium perceived friendliness $(M=1.26$, $S D=0.83), F(1,44)=21.02, p<.001, \eta_{p}^{2}=0.323$.

2) Approach Speed $(\mathrm{mm} / \mathrm{s})$ : Participants of high perceived friendliness approached the confederate faster $(M=1069.62$, $S D=254.89)$ than those of medium perceived friendliness
$(M=729.83, S D=235.05), F(1,44)=21.48, p<.001$, $\eta_{p}^{2}=0.328$.

3) Individual Distance ( $\mathrm{mm}$ ): Participants of high perceived friendliness stood closer $(M=1252.28, S D=541.78)$ to the confederate than those of medium perceived friendliness $(M=$ 2280.52, $S D=769.77), F(1,44)=28.08, p<.001, \eta_{p}^{2}=$ 0.390 .

4) Attentiveness: There was a significant relationship between the perceived friendliness and the waiting location chosen by the participants, $\chi^{2}(2, N=84)=34.69, p<.001$. Participants of low perceived friendliness tended to wait outside of the confederate's view (89.29\%), whereas participants of high perceived friendliness tended to wait within the confederate's view (89.29\%). Participants of medium perceived friendliness did not show a strong preference on locations either inside $(46.43 \%)$ or outside $(53.57 \%)$ of the confederate's view.

\section{E. Presenting Behavior}

In analyzing participants' presenting behavior, we further considered the confederate's gaze behavior. In the case of no gaze cuing, only three participants of low perceived friendliness approached the confederate to present the exhibit. This small amount of data yielded a large variance in standard error: twice as much variance as in the other cases. We, therefore, excluded this data in the following analyses.

1) Response Time (s): Without gaze cuing-Participants of high perceived friendliness responded faster $(M=4.66, S D=$ 2.47) than those of medium friendliness $(M=10.46, S D=$ 7.51), $F(1,23)=7.41, p=.012, \eta_{p}^{2}=0.244$.

Gaze cuing-The response time on average was $0.67(S D=$ $0.30), 0.69(S D=0.35)$, and $0.40(S D=0.25)$ seconds for the participants of low, medium, and high perceived friendliness, respectively. An analysis of variance found a significant main effect of the perceived friendliness, $F(2,35)=3.79, p=.032$, $\eta_{p}^{2}=0.178$. A post-hoc Tukey's HSD test further showed that participants' response time in the cases of low and medium friendliness did not differ significantly, $p=.988$; the difference between low and high perceived friendliness was marginal, $p=.095$, whereas the difference between medium and high perceived friendliness was significant, $p=.043$.

2) Approach Speed ( $\mathrm{mm} / \mathrm{s})$ : Without gaze cuingParticipants of high perceived friendliness approached the confederate to present the exhibit significantly faster $(M=935.75, S D=395.68)$ than those of medium friendliness $(M=623.39, S D=258.47), F(1,23)=5.11, p=.034$, $\eta_{p}^{2}=0.183$.

Gaze cuing - The approach speed on average was 617.00 $(S D=195.61), 852.71(S D=136.01)$, and $916.20(S D=$ 198.62) for participants of low, medium, and high perceived friendliness, respectively. A significant main effect of the perceived friendliness was found, $F(2,35)=8.85, p<.001$, $\eta_{p}^{2}=0.336$. A post-hoc Tukey's HSD test showed that the participants' approach speed between low and medium friendliness, $p<.001$, and between low and high friendliness, $p<.001$, differed significantly; however, the speed differences 
TABLE I

BEHAVIORAL PARAMETERS FOR THE ROBOT USED IN THIS WORK.

\begin{tabular}{|c|c|c|c|c|c|}
\hline & $\begin{array}{c}\text { Perceived } \\
\text { Friendliness }\end{array}$ & $\begin{array}{c}\text { Response } \\
\text { Time }\end{array}$ & $\begin{array}{c}\text { Approach } \\
\text { Speed }\end{array}$ & $\begin{array}{l}\text { Individual } \\
\text { Distance }\end{array}$ & Attentiveness \\
\hline \multirow{3}{*}{ 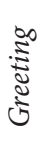 } & Low & N/A & N/A & $\mathrm{N} / \mathrm{A}$ & Invisible \\
\hline & Medium & $3.2 \mathrm{~s}$ & $0.49 \mathrm{~m} / \mathrm{s}$ & $2.5 \mathrm{~m}$ & Closest \\
\hline & High & $0.0 \mathrm{~s}$ & $0.75 \mathrm{~m} / \mathrm{s}$ & $1.5 \mathrm{~m}$ & Visible \\
\hline \multirow{3}{*}{ 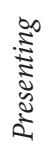 } & Low & N/A & N/A & $\mathrm{N} / \mathrm{A}$ & Invisible \\
\hline & Medium & $10.5 \mathrm{~s}$ & $0.49 \mathrm{~m} / \mathrm{s}$ & $2.5 \mathrm{~m}$ & Closest \\
\hline & High & $4.7 \mathrm{~s}$ & $0.75 \mathrm{~m} / \mathrm{s}$ & $1.5 \mathrm{~m}$ & Visible \\
\hline
\end{tabular}

between medium and high friendliness did not differ significantly, $p=.614$.

3) Individual Distance ( $\mathrm{mm})$ : Without gaze cuingParticipants of high perceived friendliness kept a shorter distance from the confederate $(M=924.88, S D=179.48)$ compared to those of medium friendliness $(M=1505.27$, $S D=662.08), F(1,23)=9.94, p=.005, \eta_{p}^{2}=0.302$.

Gaze cuing-The individual distance on average was $2251.62(S D=767.94), 1194.86(S D=238.13)$, and 1005.88 $(S D=301.93)$ for participants of low, medium, and high perceived friendliness, respectively. There was a significant main effect of the perceived friendliness, $F(2,35)=24.24$, $p<.001, \eta_{p}^{2}=0.581$. A post-hoc Tukey's HSD test showed that individual distance between low and medium friendliness, $p<.001$, and between low and high friendliness, $p<.001$, differed significantly. However, there was no significant difference found between medium and high friendliness, $p=.520$.

4) Attentiveness: After presenting the exhibit, all the participants waited at locations of the same attentiveness as those they chose to wait after greeting the confederate.

\section{F. Summary}

The above results confirmed our observations and supported the notion that people behave differently according to their relationships with and attitudes toward others [2, 10, 13, 20. 40]. Moreover, the results were consistent with the literature suggesting that greater openness and accessibility in communication signifies a closer relationship [2, 27]. Particularly, participants of higher perceived friendliness tended to respond faster, approach more quickly, maintain a shorter distance, and wait inside the confederate's view.

\section{Controlling Friendliness in a Robot}

In this section, we present the implementation of the behavioral model of friendliness in an interactive robot.

\section{A. Interactive Robot System}

A humanoid robot [34], as shown in Figure 5, was used to test the human behavioral model described in Section III. This humanoid robot is appropriate for our work for several reasons. First, its biped movements are suitable for simulating humanstyle approach behavior. Second, its humanlike appearance provides comparable interaction stimuli as those in human

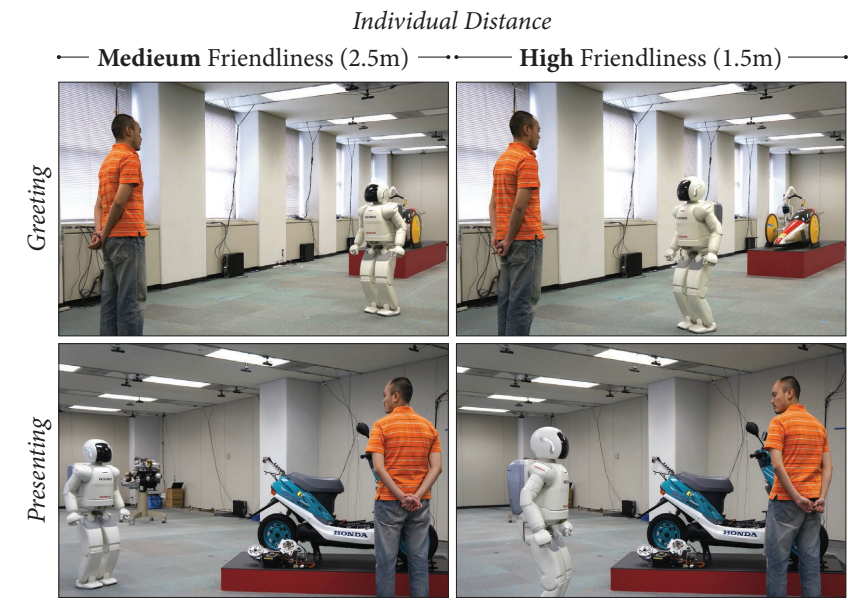

Fig. 5. The robot maintained different individual distance with visitors to convey different levels of friendliness.

interaction. Finally, the robot was designed for purposes such as assisting and engaging with people.

\section{B. Behavior Parameters}

Table II summarizes behavior parameters implemented for the robot in the evaluation study. These parameters were derived from the collected human data; however, they were adjusted for the humanoid robot used in this work.

To cope with the speed limit and system control of the robot, we adjusted the speed parameters proportionally to $0.75 \mathrm{~m} / \mathrm{s}$ and $0.49 \mathrm{~m} / \mathrm{s}$ for high and medium friendliness, respectively. Additionally, the systematic delay in the robot and controlling software was longer than the suggested human response time; therefore, we also adjusted the parameters for response time proportionally. To account for noises in the sensor data for locating the position of the visitor, $200 \mathrm{~mm}$ was added to the distance parameters, resulting in $1.5 \mathrm{~m}$ and $2.5 \mathrm{~m}$ for high and medium friendliness, respectively (Figure 5). Finally, in our pilot study with the robot, participants naturally turned to face the robot when it approached. However, the confederate was trained to face the exhibit in the data collection study. Therefore, the parameters of individual distance in the presenting behavior were set to the same as those used in the greeting behavior, reflecting a face-to-face interaction.

As suggested by the human data, an exhibition guide tended to pick a location that is either visible or invisible to the visitor while waiting. The human horizontal field-of-view on average is $200^{\circ}$ [42]. Thus, the attentiveness of the robot was defined based on a visitor's current field of view (i.e., $\pm 100^{\circ}$ from the direction of attention), as illustrated in Figure 4 Since people of medium perceived friendliness did not have a particular preference for waiting locations, the robot of medium friendliness picked a location that was closest to its current location, independent of the visibility of that location

\section{Evaluation of Human-Robot Interaction}

An evaluation in situated human-robot interaction sought to assess the validity of the behavioral model. We aim to 


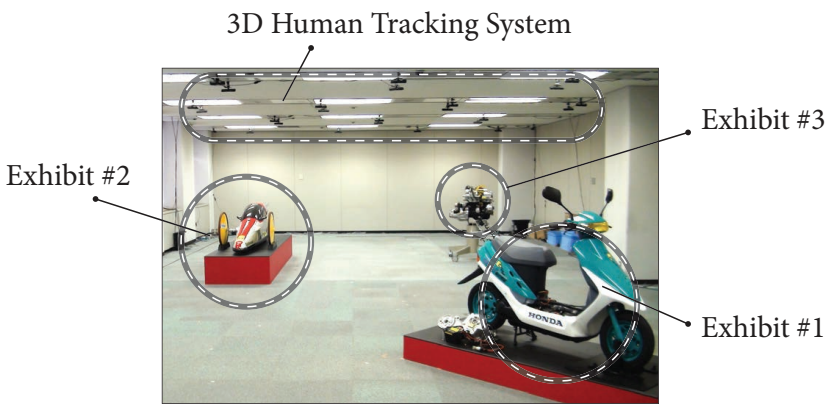

Fig. 6. The experimental setup for the museum scenario used in the humanrobot interaction study. An array of 3D human tracking system was deployed to track where the visitor was in the exhibition room.

test whether or not the designed robot behaviors of different friendliness would shape participants' perceptions of the robot's friendliness.

\section{A. Contextualization}

1) Scenario and Environment: We contextualized the evaluation in a museum scenario, shown in Figure 6. Three exhibits were set up in a 14-m by 7.7-m room. An array of $353 \mathrm{D}$ sensors (ASUS Xtion) was deployed and mounted on the ceiling of the exhibition room for the purpose of tracking people's position every 33 milliseconds. We used the tracking technique, as informed by [8], in both the evaluation study and data collection study. The integrated system, including the humanoid robot, the controlling system, and the human tracking system, ran autonomously in the evaluation study.

2) Robot Behavior: In addition to the implemented behaviors (Section IV-B), we adapted some behaviors for the evaluation of this particular experimental scenario. For the presenting behavior, we used the parameters from the case of without gaze cuing, because we did not anticipate participants gazing at the robot frequently and intentionally, given that they were instructed to act as a visitor and were informed that there was a communication robot acting as an exhibition guide.

We designed minimal speech for the robot in this evaluation study, as we wanted to focus on the effects of nonverbal behavior on conveying friendliness. The speech for greeting was a short phrase to welcome the participant. The speech for presenting was a short sentence describing the exhibit. The description for each exhibit was created to have a similar amount of information and length. The contents of speech were the same across experimental conditions.

While the human data showed that participants of low perceived friendliness did not approach to greet the confederate, more than half of the participants acknowledged the presence of the confederate by gazing toward him and provided a minimal verbal greeting. Therefore, in the evaluation, the robot of low friendliness gazed toward the participant and provided the verbal greeting, but did not approach participants.

\section{B. Experimental Conditions, Design, and Procedure}

Based on the derived model of friendliness, we developed three conditions for the evaluation study.
- Low friendliness: The robot displayed behaviors using the parameters of low perceived friendliness as shown in Table [1 We expected participants to perceive the robot to be inattentive and less willing to interact with them.

- Medium friendliness: The robot displayed behaviors using the parameters of medium perceived friendliness as shown in Table II. We expected participants to perceive the robot a bit more willing to interact with them, yet they might feel distant from the robot.

- High friendliness: The robot displayed behaviors using the parameters of high perceived friendliness as shown in Table I] We expected participants to perceive the robot to be attentive and willing to interact with them, minimizing feelings of distance with the robot.

We designed a within-subject experiment in which each participant acted as a visitor in a series of three trials, one for each condition. After receiving consent, the experimenter briefly introduced the experiment to the participant. For each trial, the participant were told a different exhibit to visit. The order of the robot's level of friendliness and the order of the exhibit to visit were counterbalanced. After each exhibit visit, the participant was asked to fill out a questionnaire regarding his or her perceptions of the robot for that particular trial. The participant repeated this procedure for the remaining trials. After all three trials, the experimenter interviewed the participant for additional comments on interaction experience.

In the beginning of each trial, the robot stood roughly at the center of the exhibition room, as if it was attending to one particular exhibit. When a participant entered the exhibition room, the robot, after the manipulated delay, acknowledged the presence of the visitor through gaze and greeted him or her. In the conditions of medium and high friendliness, the robot approached the visitor with the specified speed and stopped in front of the visitor with the specified distance. After giving a greeting, the robot walked to a waiting position, either visible or not visible to the visitor. After the specified delay, the robot approached the visitor to present the exhibit that the visitor was attending to. The behavioral parameters for approach speed and individual distance were applied to this presenting behavior. After presenting, the robot walked to a waiting position, according to the experimental condition, to wait for the visitor to finish the visit and leave the exhibition room. Note that in the condition of low friendliness, the robot did not approach to present the exhibits.

\section{Hypotheses}

In addition to the perception of friendliness, friendly behaviors might affect the feeling of liking [40]. Therefore, we developed two hypotheses regarding how participants might relate the robot's behaviors to their perceptions of the robot in terms of friendliness and likeability.

Hypothesis 1: Participants will perceive the robot exhibiting behaviors intended for high friendliness to be friendlier than the one exhibiting behaviors intended for medium friendliness and the robot exhibiting behaviors intended for medium 

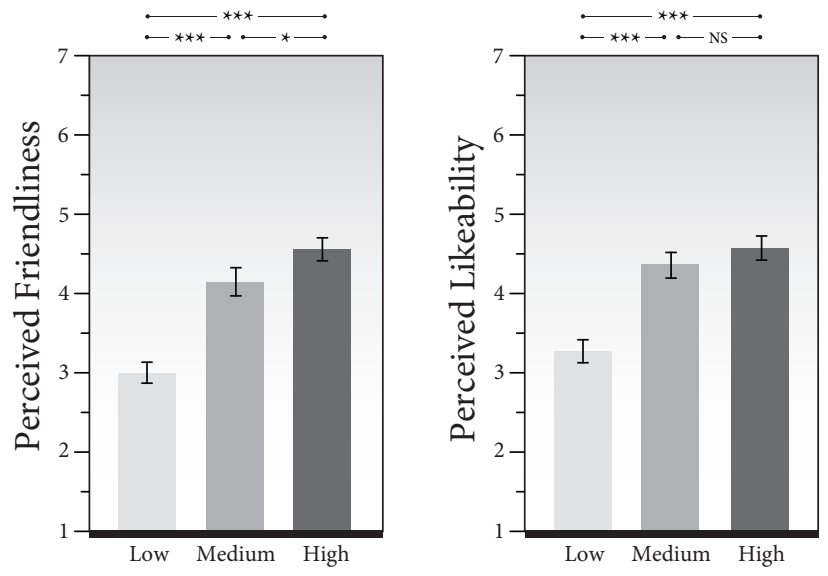

Fig. 7. Differences in the robot's behaviors elicited different perceived friendliness and likeability of the robot. $(\star),(\star \star)$, and $(\star \star \star)$ denote $p<.050$, $p<.010$, and $p<.001$, respectively.

friendliness to be friendlier than the one exhibiting behaviors intended for low friendliness.

Hypothesis 2: Participants will perceive the robot exhibiting behaviors intended for high friendliness as being more likable compared to the one exhibiting behaviors intended for medium friendliness and the robot exhibiting behaviors intended for medium friendliness as being more likable than the one exhibiting behaviors intended for low friendliness.

\section{Measurement}

To measure how friendly the robot was, we used the friendliness scales from the established personality tests [11, 12]; items were adapted to refer to robots. The resulting scale of friendliness consisted of eight items (Cronbach's $\alpha=0.88$ ). The Likeability scale (5 items, Cronbach's $\alpha=0.91$ ) [6] was used to measure how likable the robot was. All the items were in a 7-point rating scale in which 1 represented strongly disagree and 7 represented strongly agree.

\section{E. Participants}

43 participants ( 15 females and 28 males) were recruited for this evaluation study. The participants were aged from 18 to $27(M=21.81, S D=1.98)$ and reported that their familiarity with robots was on average $2.79(S D=1.60)$, using a 7point rating scale. Of all the trials, nine trials were excluded due to system failures, yielding 120 valid trials for the data analysis. All participants were native Japanese speakers and were compensated 3000 Japanese yen for their participation.

\section{F. Results}

A repeated-measures mixed-effect analysis of variance (ANOVA) was used to analyze the data. The manipulated friendliness of the robot was set as the fixed effect. We also included visited exhibit and visit order as covariates and set participant as a random effect. For all the measures, there were no significant effects due to visited exhibit and its order. Hereafter, we focus on the results caused by the manipulated friendliness. The results are summarized in Figure 7
1) Perceived Friendliness: Our first hypothesis predicted that participants would relate the robot's behaviors that were intended for different levels of friendliness to their perceptions of the robot's friendliness accordingly. Our results supported this hypothesis. Participants' ratings of the friendliness of the robot were on average $2.99(S D=0.83), 4.13(S D=1.04)$, and $4.55(S D=0.89)$ for the robot of low, medium, and high friendliness, respectively. An analysis of variance revealed that there was a main effect of the manipulated friendliness of the robot, $F(2,101)=33.30, p<.001$. Other factors and interaction effects were not significant. A post-hoc Tukey's HSD test further showed that the participants rated the robot intended to be high friendliness to be friendlier than that intended to be medium friendliness, $p=.045$, and the robot of medium friendliness to be friendlier than that of low friendliness, $p<.001$.

These results indicated that the designed behaviors operating on the designed variables successfully conveyed different levels of friendliness and influenced participants' perceptions of friendliness of the robot accordingly.

2) Perceived Likeability: Our second hypothesis predicted that the manipulated behaviors intended to show different levels of friendliness would affect how likable participants would perceive the robot. Our results partially supported this hypothesis. Participants' ratings on the likeability of the robot were on average $3.26(S D=0.91), 4.35(S D=1.07)$, and $4.56(S D=0.97)$ for the robot of low, medium, and high friendliness, respectively. An analysis of variance showed that there was a main effect of the manipulated friendliness of the robot, $F(2,101)=20.62, p<.001$. Other factors and interaction effects were not significant. A post-hoc Tukey's HSD test revealed that there were significant differences between the condition of low and high friendliness, $p<.001$, and between the condition of low and medium friendliness, $p<.001$. However, a significant difference was not found between the condition of medium and high friendliness, $p=.334$.

These results suggested that while the manipulated behaviors affected the participants' perceptions of how likable the robot was, the behaviors expressed by the robot in the conditions of medium and high friendliness might have met people's expectations for being likable to a similar degree.

3) Qualitative Results: In addition to questionnaire evaluations, participants' comments in the post-experiment interview also provided insight into how the behavioral variables shaped participants' perceptions. Several participants reported that interaction distance was related to feelings of friendliness.

\footnotetext{
"When the robot approached closer to me to present the exhibit, I felt the robot being friendly."

"I felt distant with the robot when it greeted me from a distance."
}

However, perceptions regarding the approach speed were mixed. While some participants attributed faster speed to higher friendliness, a few participants related the fast speed to a negative perception.

"The robot approaching faster created a good impression."

"The robot's approach speed made me feel pressured." 
These comments highlighted individual differences in interpreting social behaviors and indicated that there might be an appropriate range of parameter values to communicate friendliness and form intended impressions [2].

\section{DISCUSSION}

\section{A. Design Implications}

This work demonstrates how an interactive robot might use nonverbal behaviors to show varying levels of friendliness, which is integral to building rapport and the development of relationships. Our work has design implications for friendly behavior and human-robot relationships. First, behavioral variables in the temporal (i.e., response time and approach speed) and spatial (i.e., individual distance and attentiveness) domains seemed to effectively influence affective perceptions, such as feelings of friendliness. Researchers and designers may utilize both temporal and spatial variables in behavior to support affective communication, which is key in the development of relationships. Second, adaptively using different behaviors is crucial in both regulating and forming relationships. Researchers and designers need to consider variations of behavior and how to appropriately apply them in contexts that target the development of human-robot relationship. Third, there might be an appropriate value range for behavioral parameters. While increasing approach speed and shortening individual distance might seem to improve the perception of friendliness, approaching too fast or at too close distance created negative perceptions such as pressure on people. Researchers should ensure that the designed behaviors are in the acceptable ranges.

\section{B. Applicability and Generalizability}

The presented model of friendliness is subject to differences in robots, situations, and cultures. Different robotic properties might have effects on the appropriateness of the behavioral parameters. For example, while previous research suggested that distances ranging from 0.45 to 1.2 meters between individuals and robots were preferred during interaction [23], our model suggested a longer distance. However, this discrepancy might be due to the robot's appearance (e.g., humanoid or mobile robot) and method of locomotion (e.g., biped or wheelbased). Moreover, the presented model might be limited in the situation of museum setting. For instance, the behavioral variable, attentiveness, might be implemented in different ways for different settings. While it might be reasonable for a shopping assistant robot to use a user's visibility, as suggested in our model, to manipulate attentiveness, an office robot may engage in different activities to display different levels of attentiveness given that the nature of situation and spatial setup might be radically different from a museum situation. How the model might be adapted to other situations, such as classrooms, offices, or factory settings, requires further investigations. Finally, the literature in human communication has suggested cultural differences in how people might use and perceive behavior differently [3, 15, 17]. The presented model of friendliness was developed within the Japanese culture and might therefore not be applicable to other cultures.
While the presented behavioral parameters might be limited in the context, the use of the four behavioral variablesresponse time, approach speed, interpersonal distance, and attentiveness - to convey friendliness may carry over to other contexts. For example, a mobile robot serving as a shopkeeper may use these variables to show varying levels of friendliness to build rapport with its customers. Moreover, the concept of varying behaviors according to relationships with users may also be applicable to other contexts. For instance, an instructional robot may adapt its level of immediacy [27] through behaviors over time to engage its users and improve the users' perceptions of the robot and learning outcomes.

This paper presents a systematic process of how one might design adaptive behaviors for a robot to interact with humans. This process involves understanding how humans would behave in the context by collecting data of human interaction, identifying behavioral variables, and extracting parameters for those variables, building a computational model of behavior that use the variables and parameters to simulate human behavior, and evaluating the developed model in a situated human-robot interaction. In this paper, we suggest that behavioral variables in both temporal and spatial domains are effective in affective communication.

\section{Limitations and Future Work}

In addition to the limitations of generalizability discussed above, in this work we focused on nonverbal behaviors only; however, it has been established that verbal behavior is also related to the communication of interpersonal warmth [7]. Including the verbal channel to express varying levels of friendliness might magnify the effects. Moreover, we investigated the perceived friendliness of the robot using a combination of four behavioral variables. Although this setup allowed for a more holistic investigation of behaviors and social processes [40], it could not explain which variable contributed more to the perceived friendliness. Finally, future research is needed to investigate how interactive robots can gradually apply different behaviors during extended interactions with their users.

\section{CONCLUSION}

In this paper, we demonstrate how an interactive robot might express different nonverbal behaviors to shape people's perceptions of friendliness of the robot. In line with literature in human communication and relationships, the interactive robot was perceived as more friendly when showing greater openness and accessibility in communication-less response time, faster approach speed, shorter individual distance, and greater attentiveness. While showing friendly behavior plays a key role in forming positive impressions, it is important for robots to employ varying friendly behaviors to foster the development of relationships with users. This work provides insights for designing appropriately friendly robot behaviors and for enabling human-robot relationships.

\section{ACKNOWLEDGMENTS}

This research was supported by Honda R\&D. 


\section{REFERENCES}

[1] Stuart Albert and James M Dabbs Jr. Physical distance and persuasion. Journal of Personality and Social Psychology, 15(3):265, 1970.

[2] Irwin Altman and Dalmas A Taylor. Social penetration: The development of interpersonal relationships. Holt, Rinehart \& Winston, 1973.

[3] Michael Argyle. Bodily communication. International Universities Press, 1975.

[4] Michael Argyle and Mark Cook. Gaze and mutual gaze. 1976.

[5] Michael Argyle, Veronica Salter, Hilary Nicholson, Marylin Williams, and Philip Burgess. The Communication of Inferior and Superior Attitudes by Verbal and Non-verbal Signals. British journal of social and clinical psychology, 9(3):222-231, 1970.

[6] Christoph Bartneck, Dana Kulić, Elizabeth Croft, and Susana Zoghbi. Measurement instruments for the anthropomorphism, animacy, likeability, perceived intelligence, and perceived safety of robots International Journal of Social Robotics, 1(1):71-81, 2009.

[7] Marjorie A Bayes. Behavioral cues of interpersonal warmth. Journal of Consulting and Clinical Psychology, 39(2):333, 1972.

[8] D. Brscic, T. Kanda, T. Ikeda, and T. Miyashita. Person tracking in large public spaces using $3 \mathrm{~d}$ range sensors. IEEE Transaction on Human-Machine Systems, 43:522534, 2013.

[9] Wolfram Burgard, Armin B Cremers, Dieter Fox, Dirk Hähnel, Gerhard Lakemeyer, Dirk Schulz, Walter Steiner, and Sebastian Thrun. Experiences with an interactive museum tour-guide robot Artificial intelligence, 114(1): 3-55, 1999.

[10] Justine Cassell, Alastair J Gill, and Paul A Tepper. Coordination in conversation and rapport. In Proceedings of the workshop on Embodied Language Processing, pages 41-50. Association for Computational Linguistics, 2007.

[11] C Robert Cloninger, Thomas R.. Przybeck, and Dragan M.. Svrakic. The Temperament and Character Inventory (TCI): A guide to its development and use. center for psychobiology of personality, Washington University St. Louis, MO, 1994.

[12] Steven R Conn and Mark L Rieke. The 16PF fifth edition technical manual. Institute for Personality and Ability Testing Champaign, IL, 1994.

[13] Steven W Duck. Study of Acquaintance. Lexington Books, 1977.

[14] Paul Ekman and Wallace V Friesen. The repertoire of nonverbal behavior: Categories, origins, usage, and coding. Nonverbal communication, interaction, and gesture, pages 57-106, 1981.

[15] Gary W Evans and Roger B Howard. Personal space Psychological Bulletin, 80(4):334, 1973.

[16] Peter Glick, Judith A Demorest, and Carla A Hotze.
Keeping Your Distance: Group Membership, Personal Space, and Requests for Small Favors. Journal of Applied Social Psychology, 18(4):315-330, 1988.

[17] Edward Twitchell Hall and Edward T Hall. The hidden dimension. Anchor Books New York, 1969.

[18] A Paul Hare. Handbook of small group research. 1976.

[19] Stanley Heshka and Yona Nelson. Interpersonal speaking distance as a function of age, sex, and relationship Sociometry, pages 491-498, 1972.

[20] Robert A Hinde and Henry Tajfel. Towards understanding relationships, volume 18 . Academic Press London, 1979.

[21] Chien-Ming Huang and Bilge Mutlu. Modeling and Evaluating Narrative Gestures for Humanlike Robots. In Proc. RSS'13, 2013.

[22] Chien-Ming Huang and Bilge Mutlu. The repertoire of robot behavior: Enabling robots to achieve interaction goals through social behavior Journal of Human-Robot Interaction, 2(2):80-102, 2013.

[23] Helge Hüttenrauch, Kerstin Severinson Eklundh, Anders Green, and Elin Anna Topp. Investigating spatial relationships in human-robot interaction. In Intelligent Robots and Systems, 2006 IEEE/RSJ International Conference on, pages 5052-5059. IEEE, 2006.

[24] Kheng Lee Koay, Dag Sverre Syrdal, Michael L Walters, and Kerstin Dautenhahn. Living with robots: Investigating the habituation effect in participants' preferences during a longitudinal human-robot interaction study. In Robot and Human interactive Communication, 2007. ROMAN 2007. The 16th IEEE International Symposium on, pages 564-569. IEEE, 2007.

[25] Dale F Lott and Robert Sommer. Seating arrangements and status Journal of Personality and Social Psychology, 7(1p1):90, 1967.

[26] Albert Mehrabian. Relationship of attitude to seated posture, orientation, and distance Journal of Personality and Social Psychology, 10(1):26, 1968.

[27] Albert Mehrabian. Silent messages. Wadsworth, 1971.

[28] Anton S Morton. Similarity as a determinant of friendship: A multidimensional study. Technical report, DTIC Document, 1959.

[29] Jonathan Mumm and Bilge Mutlu. Human-robot proxemics: physical and psychological distancing in humanrobot interaction. In Proceedings of the 6th international conference on Human-robot interaction, pages 331-338. ACM, 2011.

[30] Illah R Nourbakhsh, Clayton Kunz, and Thomas Willeke. The mobot museum robot installations: A five year experiment. In Proceedings. 2003 IEEE/RSJ International Conference on Intelligent Robots and Systems (IROS), volume 4, pages 3636-3641. IEEE, 2003.

[31] Howard M Rosenfeld. Effect of an approval-seeking induction on interpersonal proximity. Psychological Reports, 17(1):120-122, 1965.

[32] Jamieson Schulte, Charles Rosenberg, and Sebastian Thrun. Spontaneous, short-term interaction with mobile 
robots. In Robotics and Automation, 1999. Proceedings. 1999 IEEE International Conference on, volume 1, pages 658-663. IEEE, 1999.

[33] Chris Segrin. The effects of nonverbal behavior on outcomes of compliance gaining attempts Communication Studies, 44(3-4):169-187, 1993.

[34] Satoshi Shigemi, Yuichiro Kawaguchi, Takahide Yoshiike, Koji Kawabe, and Naohide Ogawa. Development of new ASIMO Honda R AND D Technical Review, 18(1): 38, 2006.

[35] Masahiro Shiomi, Takayuki Kanda, Hiroshi Ishiguro, and Norihiro Hagita. Interactive humanoid robots for a science museum In Proceedings of the ACM conference on Human-robot interaction (HRI), pages 305-312. ACM, 2006.

[36] Patrick E Shrout and Joseph L Fleiss. Intraclass correlations: uses in assessing rater reliability Psychological bulletin, 86(2):420, 1979.

[37] Robert Sommer. Leadership and group geography Sociometry, 24(1):99-110, 1961.

[38] DS Sundaram and Cynthia Webster. The role of nonverbal communication in service encounters. Journal of Services Marketing, 14(5):378-391, 2000.

[39] Leila Takayama and Caroline Pantofaru. Influences on proxemic behaviors in human-robot interaction. In Intelligent Robots and Systems, 2009. IROS 2009. IEEE/RSJ International Conference on, pages 5495-5502. IEEE, 2009.

[40] Linda Tickle-Degnen and Robert Rosenthal. The nature of rapport and its nonverbal correlates Psychological inquiry, 1(4):285-293, 1990.

[41] Barbara Tizard. Adoption: A second chance. Free Press, 1977.

[42] Elliot B Werner and Christine G Rossi. Manual of visual fields. Churchill Livingstone New York, 1991.

[43] Frank N Willis Jr. Initial speaking distance as a function of the speakers' relationship Psychonomic Science, 1966. 